\title{
Application of $J_{2}$ Integral Method in Calculating Complicated Stress Intensity Factors on Three-dimensional Components
}

\author{
Long. $\mathrm{Li}^{1}$, Yousheng. Deng ${ }^{1 *}$, Liqing Meng ${ }^{1}$, Lingtao $\mathrm{Li}^{1}$, and Yunfang Zheng ${ }^{1}$ \\ ${ }^{1}$ College of Civil and Architectural Engineering, Xi' an University of Science and Technology, Xi' an 710054, China
}

\begin{abstract}
In this paper, a technique to determine complicated stress intensity factors on three-dimensional components, which based on the conservation law and the elementary mechanics is proposed, it only needs the geometric relationship between multiple singular stress fields from the crack section, and obtaind the relationship between the stress at different crack tips. In the expression of the stress intensity factor $\mathrm{K}, \mathrm{K}$ is proportional to the stress $\sigma$ at the crack tip, and we can get the supplementary equation of between different stress fields $\mathrm{K}$ according to the ratio of the stress at the crack tip, then use the $J$-integral method to calculate the stress intensity factors of different stress fields. In order to verify the feasibility of this method, a cracked R-fluted shells model was constructed. Under the action of the bending moment, the corner crack propagation is simulated through the reserved corner crack, and two crack tips with different stress fields are generated during the simulation. The experimental result indicates that the proposed method is effective for cracked Rfluted shells. It is also shown that the method has universal applicability for solving complex stress intensity factors on three-dimensional components.
\end{abstract}

\section{Introduction}

R-fluted shells are widely used in engineering structures, and cracks and similar cracks are common defects. The cracked R-fluted shells is a typical three-dimensional structure. Regarding infinite two-dimensional (2d) and three-dimensional (3d) elastomer crack problems, the closed solutions of SIFs can be conveniently given. However, for cracked slender R-fluted shells, which belongs to the three-dimensional (3d) problem with finite boundaries, In addition to numerical methods, it is difficult to get the analytical solutions. For cracked beams-like structures, much effort had been done to find the simple methods solving the SIFs. In 1986, a simple method for close approximation of stress intensity factor in cracked beams was proposed by Kienzler and Herrmann [1-2]. Kienzler and Herrmann assumed, without specific justification, that the strain energy release rate $G$ for crack extension is equal to that for crack widening, thereby using Irwin's G-K relation to determine the SIFs. In 1990, Bazant [3] indicated that this assumption is approximately valid within a correction factor, and claimed this can only be determined through optimum fitting of the exact solution. On the basis of previous researchs, Gao and Herrmann [4] showed that this correction factor can be obtained through simple asymptotic matching with standard limiting crack solutions in 1992. They also made some corrections and clarifications for estimation of the stress intensity factor of axial-symmetrically cracked beams. Dunn et al.[5] in 1997, based on the elementary strength theory for cracked beams forwarded by Gao and Herrmann, presented a simple, closed-form expressions for stress intensity factors for cracked I-beams subjected to a bending moment. In 2006, by estimating the SIFs of cracked Tbeams and bars, Ricci and Viola [6] presented an extension of a simple and convenient method formerly proposed by Kienzler and Herrmann. In 1998, Based on the three-dimensional (3d) conservation law and the virtual work principle, Xie et al. [7-8], proposed the $J_{2^{-}}$ integral theory, which greatly simplified the analysis of the stress intensity factor for cracked slender structures. The Xie's approach had been extended by Dotti et al. [910] as an important contribution to determine the mode-I stress intensity factor of the cracked thin-walled beam. Nobile [11-12], Kolitsch et al. [13], Livieri [14] and Alijani et al. [15], also made some efforts to utilize the simple engineering method to calculate the SIFs.

Since the $J_{2}$ integral has obvious simplicity and accuracy in solving the stress intensity factor of a threedimensional cracked shell structure, it is widely used to solve the SIF of cracked hollow shaped pipes, pipes and other engineering components[16-18]. However, the $J_{2}$ integral can only provide one equation, which may be used to solve one unknown stress intensity factor, including some cases of multiple similar cracks with the same SIFs in a cross-section [19-20]. Obviously, it is not enough for engineering applications. Nearly all the cracked beams and slender structures concerning multiple and different singular stress fields in a cross-section had been sidestepped [21]. In the present work, based on the assumption that flat cross-sections remain plane in the elementary mechanics, some supplementary equations,

\footnotetext{
* Corresponding author: dengys2009@126.com
} 
together with the $J_{2}$-integral, can be effectively used to solve the SIFs corresponding to multiple and different singular stress fields, such as the SIFs of slender R-fluted shells with periodic cracks, such as the circumferential corner cracks and inner corner cracks. It is worth noting that all the analysis and calculations of present method are within the scope of elementary strength of materials and the error is acceptable in practice.

\section{Configuration of slender R-fluted shell}

Fig. 1 gives a case of four periodic corner cracks in slender R-fluted shell under bending. There are eight crack tips with different singular stress fields, each of which has typical features of two-dimensional singular stress field in plane strain state. The $x_{2}-x_{3}$ plane in Fig. 1 is a symmetry plane for cracked shell, and all loads act in this plane; hence the bending deflections will take place in this plane also. Obviously, when $l_{\mathrm{b}}>B>t$ the deformation of the shell possesses the characteristics of the three-dimensional shell and the slender beam. Therefore, the stress intensity factors can be obtained by the conservation law and the bending theory in the material mechanics. The crack surface $A_{\mathrm{c}}$

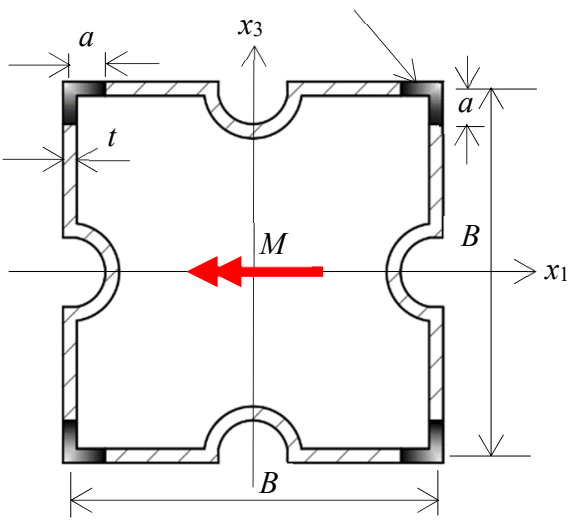

(a)

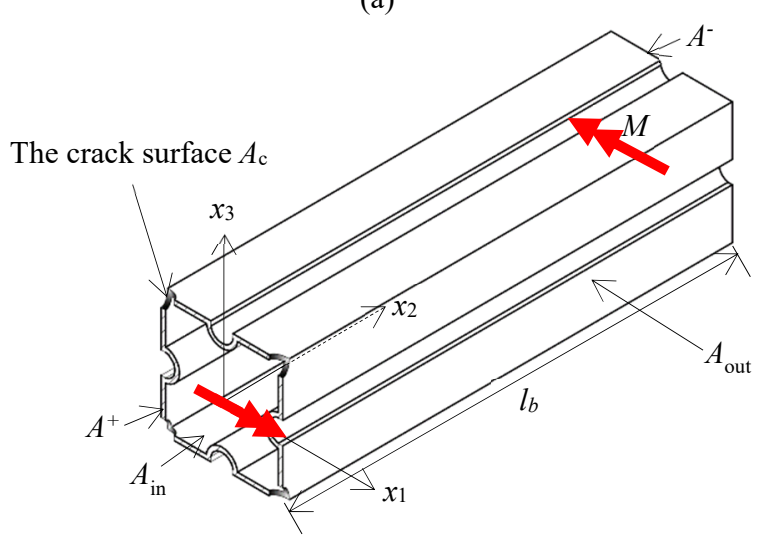

(b)

Figure 1. Periodic cracks of R-fluted shell under bending.

\section{$3 J_{2}$-integral and stress intensity factors}

Considering a three-dimensional strain field, for which the displacement vector ui depends on $x_{1}, x_{2}, x_{3}$ as shown in Fig. 2, from the conservation law, $J_{2}$-integral can be defined as [7].

$$
J_{j}=\oiint_{\Omega}\left(w \boldsymbol{n}_{j}-T_{i} u_{i, j}\right) d \Omega=0, j=1,2,3
$$

where $\Omega$ in Eq. (1) denotes an arbitrary closed surface in the solids without defects; $w$ is the strain energy density; $T_{i}$ is the stress vector acting on the outer side of $\Omega ; \boldsymbol{n}$ is the unit outward normal to $\Omega$.

For a two-dimensional deformation field. along the path $S_{\mathrm{ab}}$ and $S_{\mathrm{bc}}$, Eq. (1) yields[7]:

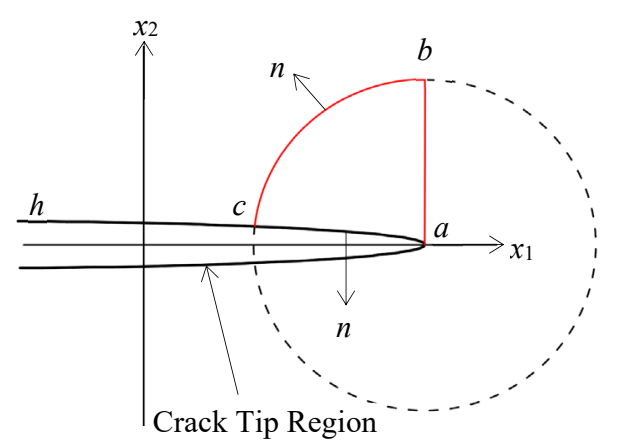

Figure 2. Integration path around crack tip region.

\section{SIFs for a R-fluted shell with circum- ferential periodic cracks under bending}

\subsection{Configuration of circumferential periodic cracks in four corners of R-fluted shell}

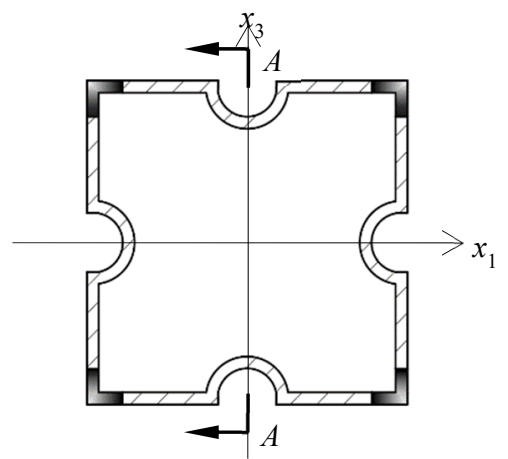

(a)

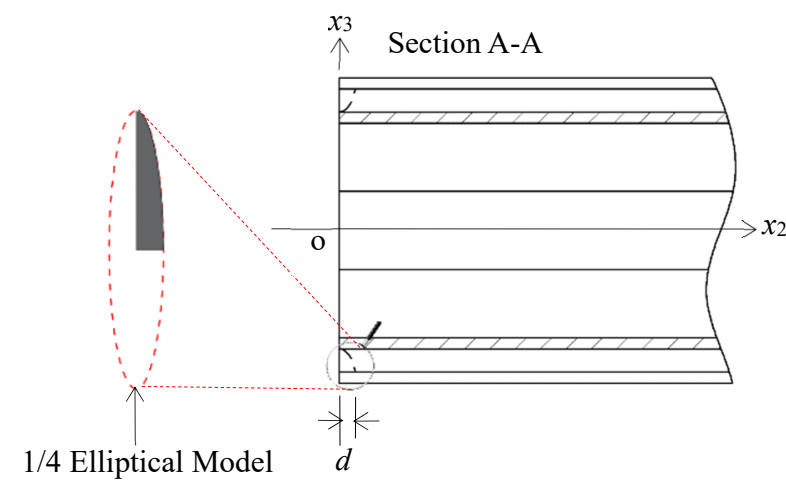

(b)

Figure 3. Elliptical modelling for cracks with $d \rightarrow 0$ 


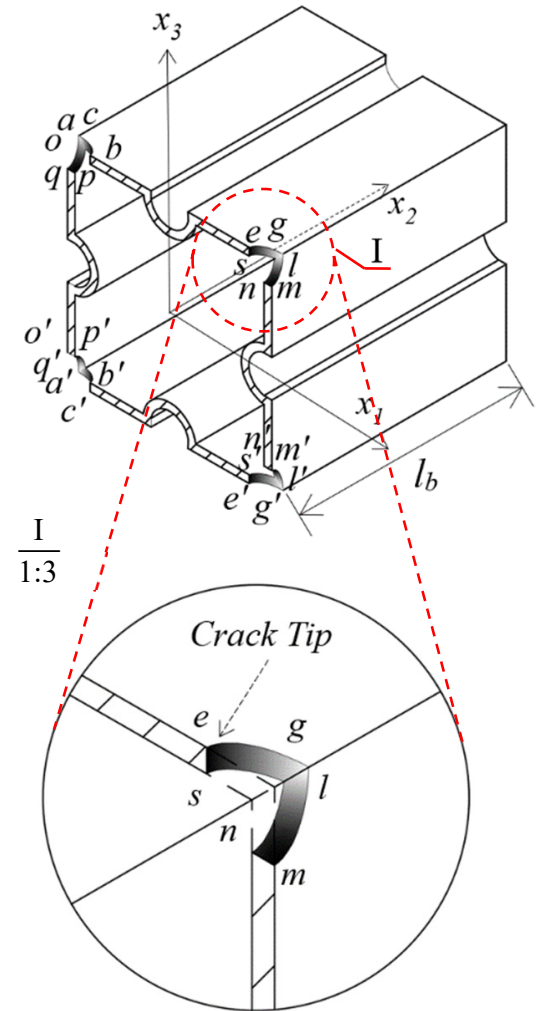

Figure 4. Local integral surfaces within the K-dominant regions.

A cracked R-fluted shell is shown in Fig. 1. The $x_{1}, x_{2}$ plane in Fig. 1 is a symmetrical plane of the shell, and all the loads act in this plane. $t$ is the thickness of the shell, $a$ is the length of the crack, where $l_{\mathrm{b}}>t, l_{\mathrm{b}}$ is the cross- section length of shell, $B$ is the cross-section width of shell, $M$ is the axial moment.

Fig. 1 shows a three-dimensional closed surface Aclosed

$$
A_{\text {closed }}=A^{+}+A^{-}+A_{\text {in }}+A_{\text {out }}+A_{c}
$$

where the symbol ' + ' denotes the cracked cross section;'-' denotes the remote uncracked cross section; Ain is the inner surface of shell; Aout is the outer surface of shell; $A_{\mathrm{c}}$ is the sum of the four crack surfaces. Since $A_{\text {in }}$ and $A_{\text {out }}$ is free surface, $T_{i}=0$ and $n_{2}=0$. following results can be found

$$
\iint_{A_{\text {in }}}\left(w n_{2}-T_{i} u_{i, 2}\right) d A=\iint_{A_{\text {out }}}\left(w n_{2}-T_{i} u_{i, 2}\right) d A=0
$$

According to beam theory, for the surfaces $A^{-}$and $A^{+}$, it is not difficult to get

$$
\begin{aligned}
& \iint_{A^{-}}\left(w n_{2}-T_{i} u_{i, 2}\right) d A=\bar{w}^{-}-M \varphi^{\prime-}=-\frac{M}{2} \varphi^{\prime-} \\
& \iint_{A^{+}}\left(w n_{2}-T_{i} u_{i, 2}\right) d A=-\left(\bar{w}^{+}-M \varphi^{\prime+}\right)=-\frac{M}{2} \varphi^{\prime+}
\end{aligned}
$$

where is the axial curvature of the R-fluted shell, is the strain energy density per unit length, $\mathrm{n}$ is unit outward normal to surface $A$ in Eqs. (4) and (5). In the remote transverse cross-section, the curvature of the axis is

$$
\varphi^{\prime-}=\frac{M}{E I}
$$

where the moment of inertia $\mathrm{I}=\mathrm{B}^{4} / 12-\pi \mathrm{D}^{4} / 64$ $2\left(\pi \mathrm{D}^{4} / 128-8 \pi \mathrm{R}^{4} / 9 \pi^{2}+\pi \mathrm{R}^{2} / 2(\mathrm{~B} / 2-4 \mathrm{R} / 3 \pi)^{2}\right)$ and $\mathrm{D}=2 \mathrm{R}$. If the crack is formed from an 1/4 ellipse with $d \rightarrow 0$ as shown in Fig. 3, the R-fluted shell can be regarded as a variable cross-section beam, and the mean curvature at the cross section becomes

$$
\phi^{+}=\lim _{d \rightarrow 0} \frac{1}{d} \int_{0}^{d} \frac{M}{E I} \frac{d x_{2}}{1-\left[\frac{8 t^{3}\left(\frac{a}{B}\right)\left(1-\left(x_{2} / d\right)^{2}\right)^{\frac{1}{2}}+48 t a\left(1-\left(x_{2} / d\right)^{2}\right)^{\frac{1}{2}}}{B^{3}}+\left(\frac{a}{B}\right) 64 t\left(1-\left(x_{2} / d\right)^{2}\right)^{\frac{3}{2}}\right] / I}=\frac{M}{E I} \gamma_{1}(a / B)
$$

Where by introducing the integral variable $\xi=x_{2} / d$,

$$
\gamma_{1}(a / B)=\int_{0}^{1} \frac{d \xi}{1-\left(\frac{a}{B}\right) \frac{\left(96 t^{3}+576 t B+768 t B^{3}\right)(1-\xi)^{\frac{1}{2}}}{B^{7}-6 \pi B^{3} R^{4}-3 \pi B^{5} R^{2}+16 R^{3} B^{4}}}
$$

Additionally, in cracked cross sectional area, there are eight asymptotic singular stress fields next to the crack tip zone, i.e., $b, b^{\prime}, e, e^{\prime}, q, q^{\prime}, m, m^{\prime}$ as shown in Fig. 4. for which the SIFs can be expressed by $K_{I b}=K_{I e}=-K_{I b^{\prime}}=-$ $K_{I e^{\prime}}, K_{I q}=K_{I m}=-K_{I q^{\prime}}=-K_{I m^{\prime}}$, The crack surface $A_{c}$ can be divided into K-dominants $\left(A_{c 1}\right)$ and free surface $\left(A_{\mathrm{c} 2}\right)$, i.e., $A_{c}=A_{c l}+A_{c 2}$., it is not difficult to get

$$
\begin{aligned}
& A_{c 1}=A_{b c}+A_{e g}+A_{l m}+A_{p q}+A_{b^{\prime} c^{\prime}}+A_{e^{\prime} g^{\prime}}+A_{l^{\prime} m^{\prime}}+A_{p^{\prime} q^{\prime}} \\
& A_{c 2}=A_{a b}+A_{e s}+A_{\mathrm{ln}}+A_{o q}+A_{a^{\prime} b^{\prime}}+A_{e^{\prime} s^{\prime}}+A_{l^{\prime} n^{\prime}}+A_{o^{\prime} q^{\prime}}
\end{aligned}
$$

Taking the three-dimensional $J_{2}$-integral over $A_{a c}$ as shown in Fig. 4 for example, by using the conservationlaw and Eqs. (1),then,

$$
\begin{aligned}
& J_{2}\left(A_{m l}\right)=\iint_{A_{m l}}\left(w n_{2}-T_{i} u_{i, 2}\right) d A=t \int_{S_{m l}}\left(w n_{2}-T_{i} u_{i, 2}\right) d s=-t \frac{\left(1-\mu^{2}\right) K_{\mathrm{Im}}^{2}}{2 \pi E} \\
& J_{2}\left(A_{e g}\right)=\iint_{A_{\text {gg }}}\left(w n_{2}-T_{i} u_{i, 2}\right) d A=t \int_{S_{e g}}\left(w n_{2}-T_{i} u_{i, 2}\right) d s=-t \frac{\left(1-\mu^{2}\right) K_{\mathrm{Ie}}^{2}}{2 \pi E}
\end{aligned}
$$

Then, from Eqs. (9)-(11), the $J_{2}$-integral over $A_{\mathrm{c}}$ becomes

$$
\begin{aligned}
& J_{2}\left(A_{c}\right)=8 J_{2}\left(A_{m l}\right)+8 J_{2}\left(A_{e g}\right)+J_{2}\left(A_{c 2}\right) \\
& =-t \frac{4\left(1-\mu^{2}\right) K_{I m}^{2}}{\pi E}-t \frac{4\left(1-\mu^{2}\right) K_{I e}^{2}}{\pi E}-J_{2}\left(A_{c 2}\right) \\
& =-t \frac{4\left(1-\mu^{2}\right)\left(K_{I m}^{2}+K_{I e}^{2}\right)}{\pi E}-\iint_{A_{c 2}} w d A
\end{aligned}
$$

Substituting the Eqs. (3)-(5) and Eq. (12) into Eq. (1), the $J_{2}$-integral over the closed surface Aclosed can be expressed as 


$$
t \frac{4\left(1-\mu^{2}\right)\left(K_{\mathrm{Im}}^{2}+K_{l e}^{2}\right)}{\pi E}+\iint_{A_{c 2}} w d A=\frac{M}{2}\left(\phi^{\prime+}-\phi^{\prime-}\right)
$$

\subsection{Supplementary equation of SIFs}

Since $J_{2}$-integral provide only one equation that contains two different SIFs, as indicated by Eq.(13), more equation is needed to solve these SIFs. For a R-fluted shells under pure bending, the distribution of the normal stress is schematically shown in Fig. 5. based on the elementary mechanics and $\sigma=\mathrm{My} / \mathrm{I}$, at the crack tips $e$ and $\mathrm{m}$ as shown in Fig. $4, \sigma_{\mathrm{e}} / \sigma_{\mathrm{m}}$ can be found as

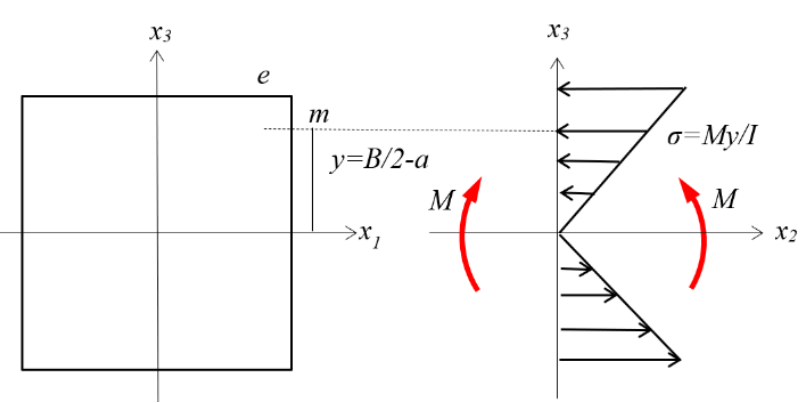

Figure 5. Distribution of the normal stress for a slender Rfluted shell under pure bending.

$$
\frac{\sigma_{e}}{\sigma_{m}}=\frac{B}{B-2 a}
$$

Referring to above equations and $K_{I}=\sigma_{0} \sqrt{\pi B}, K_{I e}$ and $K_{I m}$ can be expressed as

$$
\frac{K_{I e}}{K_{I m}}=\frac{B}{B-2 a}
$$

\subsection{Normalized SIFs}

Solving the Eqs. (13) and (15) and by using Eqs. (6) and (7), the $J_{2}$-integral over the closed surface can be expressed as

$$
\frac{4 t\left(1-\mu^{2}\right) K_{I e}^{2}}{\pi E}+\iint_{A_{c 2}} w d A=\frac{M^{2}}{2 E I}\left[\gamma_{1}(a / B)-1\right]
$$

where $\iint_{A_{c 2}} w d A$ is a small quantity and can be neglected. Hence, $f_{e}(a / B)$ and $f_{m}(a / B)$ can be found as

$$
\begin{aligned}
& f_{\mathrm{e}}(a / B)=\frac{K_{I e}}{\sigma_{0} \sqrt{\pi B}}=\left[\frac{\pi}{4 \mathrm{t}\left(1-\mu^{2}\right)}\left(\gamma_{1}(a / B)-1\right)\right]^{1 / 2} \\
& f_{m}(a / B)=\frac{K_{I m}}{\sigma_{0} \sqrt{\pi B}}=\frac{B}{B-2 a}\left[\frac{\pi}{4 \mathrm{t}\left(1-\mu^{2}\right)}\left(\gamma_{1}(a / B)-1\right)\right]^{1 / 2}
\end{aligned}
$$

where $\sigma_{0}=M B / 2 I, f_{e}(a / B)$ and $f_{m}(a / B)$ is the normalized SIFs of $K_{I e}$ and $K_{I m}$ respectively.

The mode I SIFs $K_{I e}$ and $K_{I m}$ were calculated also by using XFEM in the commercial software Abaqus, in which the C3D20 type element is employed. The slender R-fluted shell possesses both shell and beam characteristics, so the geometrical dimension of the slender R-fluted shell considered were $l_{b}=100 \mathrm{~mm}, \mathrm{~B}=$ $25 \mathrm{~mm}, t=1 \mathrm{~mm}, \mathrm{R}=5 \mathrm{~mm}, E=200 \mathrm{GPa}$ and $\mu=0.3$. The comparisons of the value of FEM and present method are as shown in Table. 1 and Table. 2 , the comparisons on the results of Eq. (17) and Eq. (18) with the finite element analysis are as shown in Fig. 6.

Table1. Comparisons of the value of FEM and Present method $\left(f_{\mathrm{e}}\right)$

\begin{tabular}{|c|c|c|c|c|c|c|c|c|c|c|}
\hline $\mathrm{a} / \mathrm{B}$ & 0.05 & 0.1 & 0.15 & 0.2 & 0.25 & 0.3 & 0.35 & 0.4 & 0.45 & 0.5 \\
\hline FEM & 0.025 & 0.056 & 0.162 & 0.270 & 0.370 & 0.520 & 1.000 & 2.300 & 6.012 & 11.040 \\
\hline Present method & 0.026 & 0.054 & 0.130 & 0.260 & 0.340 & 0.500 & 0.910 & 1.800 & 5.100 & 10.110 \\
\hline
\end{tabular}

Table2. Comparisons of the value of FEM and Present method $\left(f_{\mathrm{m}}\right)$

\begin{tabular}{|c|c|c|c|c|c|c|c|c|c|c|}
\hline $\mathrm{a} / \mathrm{B}$ & 0.05 & 0.1 & 0.15 & 0.2 & 0.25 & 0.3 & 0.35 & 0.4 & 0.45 & 0.5 \\
\hline FEM & 3.21 & 5.19 & 7.01 & 8.09 & 8.74 & 8.78 & 8.19 & 7.05 & 4.15 & - \\
\hline Present method & 3.71 & 5.76 & 7.50 & 8.19 & 8.94 & 8.58 & 8.00 & 6.85 & 3.95 & 0 \\
\hline
\end{tabular}




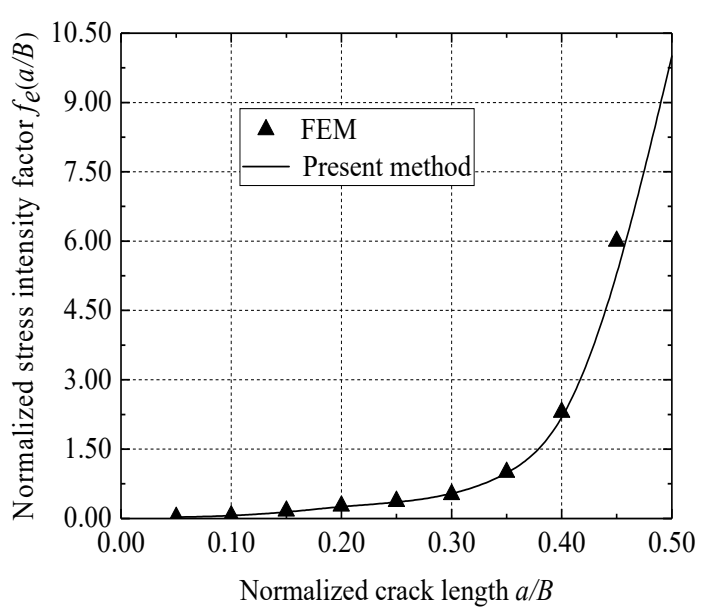

(a)

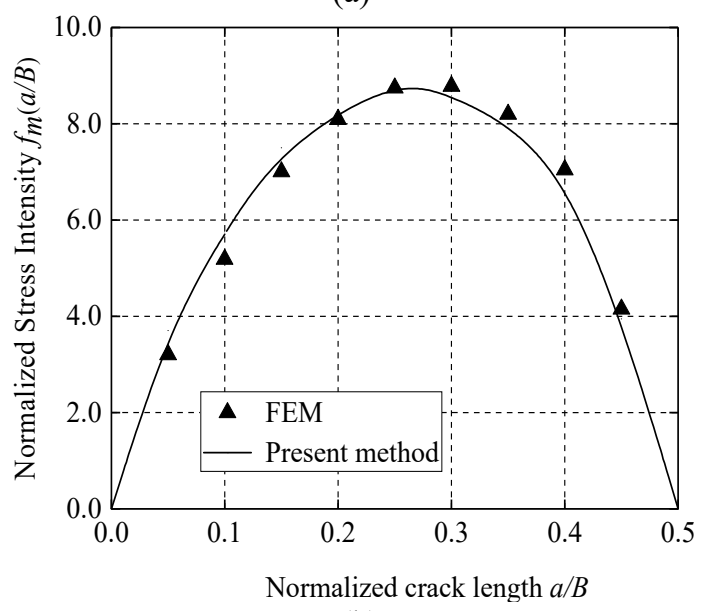

(b)

Figure 6. The normalized SIFs.

\section{Conclusions}

This paper draws the following conclusions:

(1)It is proved that $J_{2}$-integral and the assumption that plane cross-sections remain plane in the elementary mechanics is feasible for estimate the different SIFs for Rfluted shells with multiple cracks under bending.

(2)The simple relationship between different stress intensity factors can be found based on the elementary mechanics and $\sigma=\mathrm{My} / \mathrm{I}$, which provides an idea for solving the problem of stress intensity factors corresponding to complex stress fields.

(3) This method simplifies the solution of SIFs of cracked engineering structures with complex cracks and all analyses and calculations of the method are within the scope of elementary mechanics. This method is flexible and the error is acceptable in practice.

\section{Acknowledgements}

This research was financially sponsored by National Natural Science Foundation (51878554, 41672308) and Natural Science Foundation of Shaanxi (2018JZ5012).

\section{References}

1. R. Kienzler, G. Herrmann. Acta Mech, 62, 37-46. (1986)

2. G. Herrmann, H. Sosa. Eng Fract Mech, 24,889-894. (1986)

3. ZP. Bazant. Eng Fract Mech, 36, 523-525. (1990)

4. H. Gao, G. Herrmann. Eng Fract Mech, 41, 695706.(1992)

5. M. L. Dunn, W. Suwito, B. Hunter. Eng Fract Mech. 57, 609-615 (1997)

6. P. Ricci, E. Viola. Eng Fract Mech. 73, 91-111(2006)

7. Y. J. Xie, H. Xu, PN. Li. Theor Appl Fract Mech.;29, 195-203 (1998)

8. Y. J. Xie. Int J Pressure Vessels Piping. 75, 865-869. (1998)

9. FE. Dotti, VH. Cortínez, F. Reguera. Theor Appl Fract Mech. 67, 38-45. (2013)

10. VH. Cortínez, FE. Dotti. Eng Fract Mech. 110, 249257. (2013)

11. L. Nobile. Theor Appl Fract Mech. 33, 107-116. (2000)

12. L. Nobile. Theor Appl Fract Mech. 41, 137-145. (2004)

13. S. Kolitsch, HP. Gänser, R. Pippan. Theor Appl Fract Mech. 92, 167-177. (2017)

14. P. Livieri. Theor Appl Fract Mech. 84, 119-128. (2016)

15. A. Alijani, MM. Abadi, A. Darvizeh, MK. Abadi. Arch Appl Mech. 88, 875-895. (2018)

16. Y. J. Xie, X. H. Wang, Y. C. Lin. Fatigue Fract Eng M. 71, 1501-1513. (2004)

17. H. Yuan, W.J. Liu, Y.J.Xie. Eng Fract Mech. 207, 131-148. (2019)

18. W.J. Wang, Y.J. Xie. Acta Mech. 232, 619-637. (2021)

19. Y. J. Xie, X. H. Wang, Y. Y. Wang. Int J Solids Struct. 44, 4830-4844. (2007)

20. Y. J. Xie. Int J Solids Struct. 37, 5189-5201. (2000)

21. Y. Z Chen. Int J Pressure Vessels Piping. 76, 49-53. (1999) 